\title{
Activation of Melatonin Receptors Reduces Relapse-Like Alcohol Consumption
}

\author{
Valentina Vengeliene*,', Hamid R Noori' and Rainer Spanagel' \\ 'Institute of Psychopharmacology, Central Institute of Mental Health, Faculty of Medicine Mannheim, University of Heidelberg, Mannheim, \\ Germany
}

\begin{abstract}
Melatonin is an endogenous synchronizer of biological rhythms and a modulator of physiological functions and behaviors of all mammals. Reduced levels of melatonin and a delay of its nocturnal peak concentration have been found in alcohol-dependent patients and rats. Here we investigated whether the melatonergic system is a novel target to treat alcohol addiction. Male Wistar rats were subjected to long-term voluntary alcohol consumption with repeated abstinence phases. Circadian drinking rhythmicity and patterns were registered with high temporal resolution by a drinkometer system and analyzed by Fourier analysis. We examined potential antirelapse effect of the novel antidepressant drug agomelatine. Given that agomelatine is a potent MTI and MT2 receptor agonist and a 5- $\mathrm{HT}_{2 \mathrm{C}}$ antagonist we also tested the effects of melatonin itself and the 5- $\mathrm{HT}_{2 C}$ antagonist SB242084. All drugs reduced relapse-like drinking. Agomelatine and melatonin administered at the end of the light phase led to very similar changes on all measures of the post-abstinence drinking behavior, suggesting that effects of agomelatine on relapse-like behavior are mostly driven by its melatonergic activity. Both drugs caused a clear phase advance in the diurnal drinking pattern when compared with the control vehicle-treated group and a reduced frequency of approaches to alcohol bottles. Melatonin given at the onset of the light phase had no effect on the circadian phase and very small effects on alcohol consumption. We conclude that targeting the melatonergic system in alcohol-dependent individuals can induce a circadian phase advance, which may restore normal sleep architecture and reduce relapse behavior.

Neuropsychopharmacology (20I5) 40, 2897-2906; doi:I0.1038/npp.20I5.I43; published online 10 June 2015
\end{abstract}

\section{INTRODUCTION}

Most physiological functions of the body as well as certain behavioral patterns are synchronized to the presence/absence of the daylight. One of the major players responsible for the control of our daily rhythms is melatonin. It is a hormone generally synthesized in the pineal gland during the dark phase of the photoperiod (Lynch et al, 1975). Fluctuating levels of melatonin tunes cellular activity throughout the body to the actual time-of-day. High melatonin levels reinforce physiological functions and behaviors associated with the darkness, whereas low levels have the opposite effect.

Research on the interaction between alcohol and circadian rhythms started several decades ago. This interaction is bidirectional. On one hand, behavioral and physiological responses to alcohol as well as alcohol preference depend on the time-of-day (Brick et al, 1984). Rats maintained in total darkness drink more alcohol than those kept under constant illumination (Geller 1971), and an animal model called 'drinking in the dark' is in fact based on the timing of alcohol

*Correspondence: Dr $\vee$ Vengeliene, Institute of Psychopharmacology, Central Institute of Mental Health $(\mathrm{CIMH})$, Medical Faculty Mannheim/University of Heidelberg, J5, Mannheim, 68159, Germany, Tel: +49 621 |703 6261, Fax: +49621 |703 6255,

E-mail: valentina.vengeliene@zi-mannheim.de

Received 6 February 2015; revised 24 April 2015; accepted 15 May 2015; accepted article preview online 21 May 2015 exposure (Rhodes et al, 2005). On the other hand, alcohol drinking alters the circadian profile of the melatonin production and disturbed circadian rhythms were found in both alcohol-dependent patients and alcohol drinking rats (Kühlwein et al, 2003; Peres et al, 2011). Melatonin produces its effects through two melatonin receptors-MT1 and MT2 (von Gall et al, 2002). The MT1 receptor protein has been found in several brain regions known to have a crucial role in mediating the effects of drugs and addictive behavior, such as prefrontal cortex, striatum, nucleus accumbens, amygdala, and hippocampus (Uz et al, 2005; Noori et al, 2012).

The aim of our study was to investigate whether the melatonin system could be used as a target to treat alcohol addiction. For this purpose we used male Wistar rats subjected to long-term voluntary alcohol consumption in a four-bottle procedure, repeatedly interrupted with the abstinence phases. In rats that had long-term voluntary access to alcohol followed by deprivation for several weeks, the re-presentation of alcohol leads to relapse-like drinkinga temporal increase in alcohol intake over the baseline drinking. This robust phenomenon is called the alcohol deprivation effect (ADE; Sinclair and Senter, 1967; Spanagel and Hölter, 1999). Our previous research has demonstrated that alcohol consumption during these post-abstinence drinking days is characterized by an increased frequency of approaches to the alcohol bottles, which was interpreted as an increased alcohol 'wanting' (Vengeliene et al, 2013). The 
change in the temporal distribution of the alcohol consumption is associated with the loss of normal diurnal behavior, which may possibly be interpreted as development of compulsive alcohol drinking and addictive behavior. It should be noted that although the ADE provides a suitable model to study the impact of alcohol re-exposure on relapselike behavior (Lê and Shaham, 2002), it mimics only some aspects of human relapse. Transitory nature of the elevated post-abstinence alcohol consumption in rats is one characteristic that distinguish relapse drinking in rats from that seen in human alcoholics. Nevertheless, clinically effective antirelapse drugs acamprosate and naltrexone were shown to effectively reduce the ADE in rats demonstrating predictive value of this animal model (Spanagel, 2009). In recent years, the ADE animal model has become widely used for examining the efficacy of pharmacological agents in preventing compulsive alcohol consumption and relapse (Vengeliene et al, 2008; 2014).

In order to assess the role of the melatonin system in alcohol relapse we studied the effects of agomelatine, a potent MT1 and MT2 agonist, which also displays antagonistic activity on $5-\mathrm{HT}_{2 \mathrm{C}}$ receptors. The affinity of this drug for other 5-HT receptors is low (Millan et al, 2003). Two further drug treatments were included in our study to clarify the contribution of $\mathrm{MT} 1 / \mathrm{MT} 2$ receptors and $5-\mathrm{HT}_{2 \mathrm{C}}$ receptors in mediating the effects of agomelatine on relapse-like drinking and its action on circadian rhythm. For this purpose a high dose of melatonin was administered repeatedly either at the end or at the onset of the light phase. We also tested the effect of 5- $\mathrm{HT}_{2 \mathrm{C}}$ antagonist SB242084. Both agomelatine and SB242084 were administered at the end of the light phase. To characterize temporal characteristics of drinking behavior we registered drinking patterns with a high temporal resolution using a drinkometer system (Vengeliene et al, 2013).

\section{MATERIALS AND METHODS}

\section{Animals}

Fifty-six 2-month-old male Wistar rats (from our own breeding colony at the CIMH, Mannheim, Germany) were used. All animals were housed individually in standard rat cages (Type-III; Ehret, Emmendingen, Germany) under a 12/12-h artificial light/dark cycle (lights on at 0700 hours). Room temperature was kept constant (temperature: $22 \pm 1^{\circ} \mathrm{C}$, humidity: $55 \pm 5 \%$ ). Standard laboratory rat food (Ssniff, Soest, Germany) and tap water were provided ad libitum throughout the experimental period. Body weights were measured weekly. All experimental procedures were approved by the Committee on Animal Care and Use (Regierungspräsidium Karlsruhe), and carried out in accordance with the local Animal Welfare Act and the European Communities Council Directive of 24 November 1986 (86/609/EEC).

\section{Drugs}

Ethanol drinking solutions were prepared from 96\% ethanol (Sigma-Aldrich, Taufkirchen, Germany) and then diluted with tap water. Melatonin (Enzo Life Sciences, Lörrach, Germany) was suspended in $0.5 \%$ methylcellulose (Sigma-Aldrich, Taufkirchen, Germany) and injected as a volume of $3 \mathrm{ml} / \mathrm{kg}$ intraperitoneally (IP). Agomelatine (generously provided by Abbvie, Ludwigshafen, Germany) was suspended in $1 \%$ hydroxypropyl methylcellulose (SigmaAldrich, Taufkirchen, Germany) and injected as a volume of $1 \mathrm{ml} / \mathrm{kg}$ IP. SB242084 (generously provided by Abbvie, Ludwigshafen, Germany) was dissolved in water for injections (aqua ad iniectabilia, Braun, Melsungen AG, Germany) and injected as a volume of $1 \mathrm{ml} / \mathrm{kg}$ IP. Control animals received an equal volume of respective vehicle.

\section{Drinkometer System}

The drinkometer system has been developed together with TSE Systems (Bad Homburg, Germany). It enables continuous long-term monitoring of liquid consumption by amount and time in a standard rat home cage (Eurostandard Type III). The system is equipped with four drinking stations to allow liquid choice. The drinking station consists of a glass vessel containing the liquid and a high-precision sensor for weighing the amount of liquid removed from the glass vessel. Spillage and evaporation are minimized by using special bottle caps. Monitoring of all drinking stations is carried out by a computer. The system features ultrahigh resolution-down to $0.01 \mathrm{~g}$. The whole system is mounted to a custom-made freeswinging steel frame in order to avoid any environmental disturbances. The drinkometer system measures the weight of a vessel in $200 \mathrm{~ms}$ steps and saves it in 1-s steps. The normal sampling can be set with minimum 1 min intervals. For the present study, sampling was performed at $5 \mathrm{~min}$ intervals.

\section{Long-Term Ethanol Consumption with Repeated Deprivation Phases}

After 2 weeks of habituation to the animal room, rats were given ad libitum access to tap water and 5, 10, and 20\% ethanol solutions (v/v). The first 2-week deprivation period was introduced after 8 weeks of continuous ethanol availability. After this deprivation period, rats were given access to ethanol again. This access was further interrupted repeatedly with deprivation periods in a random manner (ie, the duration of following drinking and deprivation phases was irregular: approximately $4-6$ and $2-3$ weeks, respectively). The long-term voluntary ethanol drinking procedure including all deprivation phases lasted a total of $\sim 1$ year.

\section{Pharmacological Studies}

The pharmacological studies started at the end of the sixth ethanol deprivation phase. In order to study the effects of drug treatment on the expression on $\mathrm{ADE}$, rats were divided into groups $(n=7-10$, see the figures for exact number of animals used in each group) in such way that the mean baseline total ethanol intake between vehicle and drug-treated group, as well as the intake of every solution separately (ie, water, $5 \%, 10 \%$, and $20 \%$ ethanol), were matched. Baseline drinking was monitored for 6 days. After the last day of baseline measurement, the ethanol bottles were removed from the cages, leaving the animals with free access to food and water for 2-3 weeks. Thereafter, each animal was subjected to a total of three daily injections of either vehicle or compounds: agomelatine $(30 \mathrm{mg} / \mathrm{kg})$, melatonin $(50 \mathrm{mg} / \mathrm{kg})$, and SB242084 $(3 \mathrm{mg} / \mathrm{kg})$. The doses of these drugs were chosen based on our pilot studies. Melatonin was 
administered either at the end of light phase (at ZT12) or at the onset of the light phase (at ZT0), whereas agomelatine and SB242084 were given only at ZT12 (Supplementary Figure 1). The ethanol bottles were reintroduced immediately after the second injection and the drinking from each bottle was measured each $5 \mathrm{~min}$ for the following 6 days. Total ethanol intake (in $\mathrm{g}$ of pure ethanol per $\mathrm{kg}$ of body weight per 24-h day) was calculated from this data. Each rat's body weight was recorded $24 \mathrm{~h}$ before the first injection and $12 \mathrm{~h}$ after the last injection.

\section{Home Cage Locomotor Activity Measurements by the E-Motion System}

In order to test for any sedative effects resulting from the drug treatment, home cage locomotor activity was monitored by use of an infrared sensor connected to a recording and data storing system (Mouse-E-Motion, Infra-e-motion, Henstedt-Ulzburg, Germany). A Mouse-E-Motion device was placed above each cage ( $30 \mathrm{~cm}$ from the bottom) so that the rat could be detected at any position inside the cage. The device was sampling every second, whether the rat was moving or not. The sensor could detect body movement of the rat of at least $1.5 \mathrm{~cm}$ from one sample point to the successive one. The locomotor activity device was mounted above the home cage 3 days before drug treatment procedure and was continued for four more post-treatment days. The percentage of each rat's locomotor activity during and after treatment days was calculated by using the 'before treatment' activity data as a reference. The data measured by each Mouse-E-Motion device were downloaded into a personal computer and processed with Microsoft Excel.

\section{Data Analysis}

Data on total daily ethanol intake, water intake, and locomotor activity were analyzed using a two-way analysis of variance (ANOVA) with repeated measures (factors: between subjects-treatment group (vehicle $v s$ drug), and within subjects-pre- and post-abstinence days). For the analysis of locomotor activity, only the data from the dark phases were used. Whenever significant differences were found, post hoc Student Newman-Keuls test was performed. Data analysis regarding the effects of treatment on the change in the rat body weight was performed using independent two-tailed $t$-test. The chosen level of significance was $P<0.05$.

To investigate the effects of different drugs on the drinking patterns of animals, we used an analogous approach to our previous study (Perreau-Lenz et al, 2012; Vengeliene et al, 2013). We utilized Fourier analysis to characterize the recurrent drinking events within the drinkometer data sets and pursued different strategies to capture all critical aspects of drug effects. Ethanol- and water-drinking pattern analysis was performed using data of the first six post-abstinence drinking days (see Figures 1-4). This analysis enabled us to analyze two specific components of the drinking behavior: (i) the abstinence-induced changes of drinking patterns; (ii) treatment-induced alterations of drinking patterns and shift of circadian phase. A separate analysis was performed using data of the last 3 baseline drinking days and the first $\mathrm{ADE}$ day, when animals were receiving drug treatment. During the first post-abstinence drinking day animals show the most pronounced relapse-like drinking behavior (Vengeliene et al, 2013). The Fourier analysis provides a function $f(t)=\sum_{n=0}^{\infty}\left[a_{n} \cos \left(\frac{2 \pi n t}{L}\right)+b_{n} \sin \left(\frac{2 \pi n t}{L}\right)\right]$ with the maximum likelihood to describe the amount of water drinking or drinking of ethanol mixtures in the drinkometer system during the measurement interval $L$. On the basis of the frequencies $2 \pi n / L$ and the Fourier coefficients $a_{n}=\frac{2}{L} \int_{0}^{L} f(t) \cos \left(\frac{2 \pi n t}{L}\right) \mathrm{d} t$, respectively $b_{n}=\frac{2}{L} \int_{0}^{L} f(t) \sin \left(\frac{2 \pi n t}{L}\right) \mathrm{d} t$, this approach provides approximate measures for drinking frequencies, drinking peak times, and peak intake in control groups and enabled us to compare the drinking behavior of experimental groups based on these properties. In MATLAB we used the maximum likelihood estimation (MLE) to determine the Fourier coefficients of our model based on the drinking data sets. Using 5 -min drinking data for each ethanol mixture $(5,10$, and $20 \%)$ and water, the MLE method suggests two sets of Fourier parameters $\left\{a_{n}, b_{n}\right\}$ respectively $\left\{\tilde{a}_{n}, \tilde{b}_{n}\right\}$ and the frequencies $2 \pi n / L$ for Fourier representations of averaged drinking amounts $f(t)$ for the control group respectively $\tilde{f}(t)$ for the group of animals treated with the drug. We analyzed the differences between two groups based on the direct coefficient comparisons $D_{n}^{a}=\left\|a_{n}-\tilde{a}_{n}\right\|, D_{n}^{b}=\left\|b_{n}-\tilde{b}_{n}\right\|$, and by the differences of the area under the curves of each model function $A(c)=\left\|\int_{0}^{L} f(t) d t-\int_{0}^{L} \tilde{f}(t)\right\|$ for each ethanol concentration $c=\{0,5 \%, 10 \%, 20 \%\}$. Phase angles of the Fourier series for each case were calculated as $\varphi_{n}=\tan ^{-1}\left(-b_{n} / a_{n}\right)$. The phase shifts for each post-abstinence day were determined then as $D_{n}^{\varphi}=\tilde{\varphi}_{n}-\varphi_{n}$. The parameters $D_{n}^{a}, D_{n}^{b}$ and $D_{n}^{\varphi}$ have been applied to classify significant differences between the vehicle and drug administration throughout this study. We analyzed the Fourier coefficient variations using a one-way ANOVA. The chosen level of significance was $P<0.05$. Specifically, the parameters intake $\left(a_{0}\right)$ and frequency $(2 \pi n / L)$ were compared within and between the vehicle- and drug-treated animals, describing the approximate mean of water/ethanol intake and the number of maximal intake peak occurrences in $1 \mathrm{~h}$, respectively.

\section{RESULTS}

\section{Increased 'Wanting' for Higher Concentrated Ethanol Solutions During the ADE}

Following the reintroduction of ethanol solutions after a period of abstinence, the vehicle-treated animal groups showed a typical increase in ethanol consumption (from Figures 1a-4a), while water intake dropped down below baseline drinking levels (from Figures $1 \mathrm{c}-4 \mathrm{c}$ ), indicating the occurrence of an ADE. Subsequent Fourier analysis of 5-min drinking data blocks compared the last 3 baseline days with the first ADE day (Table 1). A significant increase in the frequency of approaches to all three ethanol solutions during the first ADE day was measured in control animals. On average there was 6 -fold increase over the baseline for $5 \%$ ethanol, 24-fold increase for $10 \%$ ethanol, and 32-fold increase for $20 \%$ ethanol, whereas frequency of approaches to the water bottle did not change, indicating an increased 'wanting', especially for higher concentrated ethanol 
a

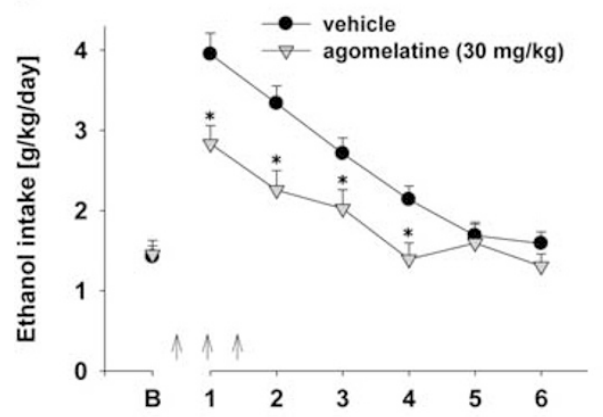

C

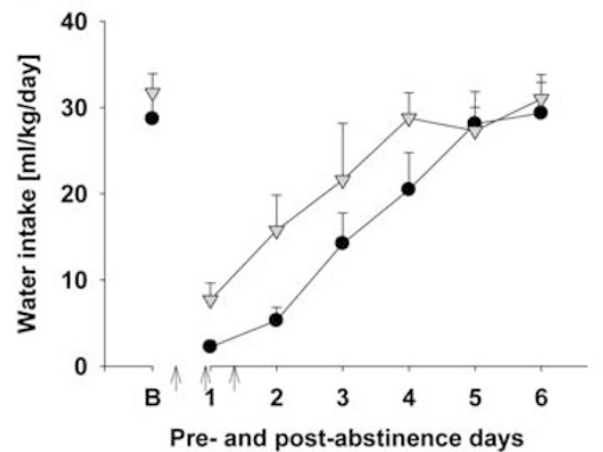

b

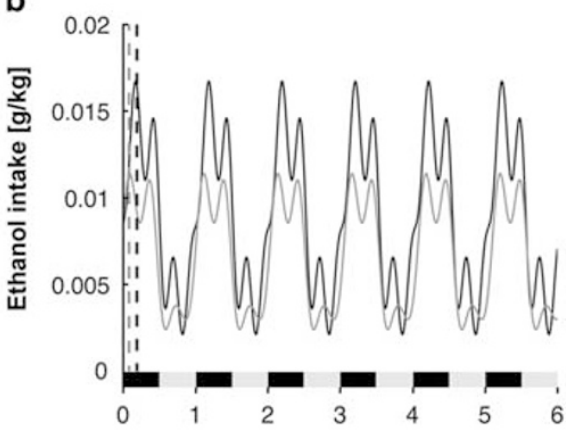

d

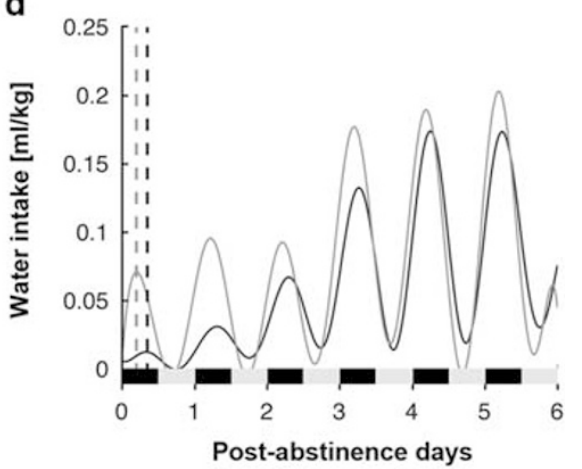

Figure I Total daily ethanol intake ( $\mathrm{g} / \mathrm{kg}$ per day) before and after an alcohol deprivation period of 2 weeks (a) and the Fourier representations of the averaged 5-min ethanol $(\mathrm{g} / \mathrm{kg}$ ) intake during the first six post-abstinence drinking days (b). Total daily water intake ( $\mathrm{ml} / \mathrm{kg}$ per day) before and after an alcohol deprivation period of 2 weeks (c) and the Fourier representations of the averaged 5-min water $(\mathrm{ml} / \mathrm{kg})$ intake during the first six post-abstinence drinking days (d). Arrows indicate the administration of either vehicle $(n=10)$ or $30 \mathrm{mg} / \mathrm{kg}$ of agomelatine $(n=10)$. Drug administration was performed at ZT I2 (ie, at the end of the light phase). The last six days measurements of ethanol and water intake is given as baseline drinking - B. Data are presented as means \pm SEM *indicates significant differences from the vehicle treatment group, $P<0.05$. In (b) and (d) the vehicle-treated animal group is marked as a black line and the agomelatine-treated animal group is marked as a gray line. Vertical dashed lines mark the peak fluid consumption within the first $24 \mathrm{~h}$ in vehicle (black) and agomelatine (gray) treated animal groups. The black and gray bars on the $\mathrm{x}$-axis indicate the dark and light phases, respectively.

Table I The Model Parameters Derived from the Fourier Coefficients Describe the Mean Water/Ethanol Intake During 5-min Intervals (intake: in $\mathrm{ml} / \mathrm{kg}$ of body weight for water, and in grams of pure alcohol per $\mathrm{kg}$ of body weight for each ethanol solution) and the Number of Maximal Intake Peak Occurrences in I h (frequency)

\begin{tabular}{|c|c|c|c|c|c|}
\hline Treatment & Fourier coefficients & Water & $5 \%$ EtOH & I0\% EtOH & $20 \%$ EtOH \\
\hline \multicolumn{6}{|l|}{ Baseline } \\
\hline & $\begin{array}{l}\text { Intake } \\
\text { Frequency }\end{array}$ & $\begin{array}{l}0.108 \\
0.044\end{array}$ & $\begin{array}{l}0.001 \\
0.044\end{array}$ & $\begin{array}{l}0.003 \\
0.043\end{array}$ & $\begin{array}{l}0.001 \\
0.044\end{array}$ \\
\hline \multicolumn{6}{|l|}{$A D E$, day 1} \\
\hline Control & $\begin{array}{l}\text { Intake } \\
\text { Frequency }\end{array}$ & $\begin{array}{c}0.009^{+} \\
0.042\end{array}$ & $\begin{array}{l}0.004^{+} \\
0.284^{+}\end{array}$ & $\begin{array}{l}0.004 \\
1.030^{+}\end{array}$ & $\begin{array}{l}0.001 \\
1.419^{+}\end{array}$ \\
\hline Melatonin/ ZTI2 & $\begin{array}{l}\text { Intake } \\
\text { Frequency }\end{array}$ & $\begin{array}{l}0.016^{*,+} \\
0.873^{*,+}\end{array}$ & $\begin{array}{l}0.004^{+} \\
0.048^{*}\end{array}$ & $\begin{array}{c}0.005 \\
0.140^{*,+}\end{array}$ & $\begin{array}{l}0.002 \\
0.044 *\end{array}$ \\
\hline Melatonin/ ZTO & $\begin{array}{l}\text { Intake } \\
\text { Frequency }\end{array}$ & $\begin{array}{l}0.075 * \\
0.051\end{array}$ & $\begin{array}{l}0.002^{+} \\
0.042^{*}\end{array}$ & $\begin{array}{l}0.001^{*,+}+ \\
0.252^{*++}\end{array}$ & $\begin{array}{c}0.00 \text { I } \\
0.272 *^{*+}\end{array}$ \\
\hline SB242084/ ZTI2 & $\begin{array}{l}\text { Intake } \\
\text { Frequency }\end{array}$ & $\begin{array}{c}0.065^{*},+ \\
0.054\end{array}$ & $\begin{array}{l}0.002^{*,+} \\
0.064^{*},+\end{array}$ & $\begin{array}{c}0.005 \\
0.00{ }^{*},+\end{array}$ & $\begin{array}{l}0.004^{*,+} \\
0.213^{*,+}\end{array}$ \\
\hline
\end{tabular}

The table shows the Fourier coefficients calculated for the last 3 days of baseline drinking (Baseline) and the first post-abstinence day (ADE, day I) in vehicle-treated rats and drug-treated rat groups (note: during the baseline and vehicle treatment conditions all groups of rats had nearly identical Fourier coefficients, therefore average coefficients for all control groups are presented in the table). ZTI2 indicates that drugs were administered at the end of the light phase, ZT0 indicates that drugs were administered at the onset of the light phase, ${ }^{*}$ indicates significant difference from the vehicle control group, ${ }^{+}$indicates significant differences from the baseline condition, $P<0.05$. 
solutions. The average amount of ethanol consumed during a drinking approach (measured as an average intake during a 5-min interval) in control animals was either slightly increased or remained similar to that measured under the baseline drinking conditions. Contrary, average 5-min water intake during the first $\mathrm{ADE}$ day was markedly declined (down to $\sim 10 \%$ of baseline intake).

\section{Agomelatine Reduces 'Wanting' and Relapse-Like Drinking}

All four drug treatments reduced the frequency of approaches to the ethanol bottles. The strongest effect on drinking frequency was found in the agomelatine treatment group (there was still a twofold increase in frequency of approaches over the baseline to both 10 and $20 \%$ ethanol solutions) and in the melatonin treatment at the ZT12 group (only 10\% ethanol drinking frequency was still threefold increased) (Table 1). All four drug treatments had no or very little effect on average 5-min ethanol consumption (Table 1).

Analysis of the data collected from the agomelatine treatment experiment revealed a general significant increase in total daily ethanol intake after a deprivation phase as compared with basal drinking in the vehicle-treated control group (factor day: $\mathrm{F}(6,108)=46.8, P<0.0001$ ). Administration of agomelatine caused a significant reduction in ethanol intake as compared with that in the vehicle rat group (factor treatment group: $\mathrm{F}(1,18)=8.2, \quad P<0.05$ and treatment group $\times$ day interaction effect: $\mathrm{F}(6,104)=4.4, P<0.001)$, although a subsequent post hoc analysis revealed that total ethanol consumption was still significantly increased over the baseline drinking levels during the first two postabstinence days (Figure 1a). Fourier analysis of the agomelatine treatment data demonstrated that this treatment shifted the peak of ethanol consumption to earlier in the night, which was confirmed by the phase-shift calculations, showing significant overall phase advance (by $1 \mathrm{~h}$ and 14 min, $P<0.01)$ in agomelatine-treated rats as compared with vehicle-treated rats (Figure 1b, Table 2). During the first post-abstinence days, water intake was slightly but not significantly increased in the agomelatine-treated group (factor treatment group and treatment group $\times$ day interaction effect $P=0.14$ and $P=0.37$, respectively; Figure $1 \mathrm{c}$ ). Similarly to ethanol, the water drinking peak shifted to earlier in the night by agomelatine treatment (by $1 \mathrm{~h} 57 \mathrm{~min}$, $P<0.001)$ compared with control vehicle-treated rats (Figure 1d,Table 2). Neither locomotor activity nor body weight was significantly changed by this treatment (factor treatment group: $P=0.17$ and $P=0.09$ for locomotor activity and body weight, respectively; data not shown).

\section{The Effect of Agomelatine on Relapse-Like Behavior is Mainly Driven by Its Melatonergic Activity}

Similarly to the agomelatine experiment, during the melatonin treatment at ZT12 there was a general significant increase in ethanol intake when compared with basal drinking (factor day: $\mathrm{F}(6,84)=28.7, P<0.0001$ ). However, this increase was seen only in the vehicle-treated animal group (Figure 2a). In melatonin-treated animals, total ethanol intake during the first post-deprivation day was similar to that seen during baseline drinking and even
Table 2 Fourier Analysis Calculations of Average Circadian Phase Shift (min) During Six post-abstinence Drinking Days.

\begin{tabular}{lll}
\hline Treatment & Water & Ethanol \\
\hline Agomelatine ZTI2 & $-116.79 *$ & $-73.85^{*}$ \\
Melatonin ZTI2 & $-191.39 *$ & $-32 *$ \\
Melatonin ZT0 & -2.6 & +0.96 \\
SB242084 ZTI2 & $-146.2 *$ & +22.09 \\
\hline
\end{tabular}

The data show average advancement $(-)$ or delay $(+)$ of the daily peak of water and ethanol consumption in drug-treated rats as compared with vehicle-treated rats. ZTI2 indicates that drugs were administered at the end of the light phase, ZTO indicates that drugs were administered at the onset of the light phase, *indicates significant difference from the vehicle control group.

dropped below baseline levels from the second day onward. A two-way ANOVA displayed a significant difference in ethanol intake between vehicle- and melatonin-treated animal groups (factor treatment group: $\mathrm{F}(1,14)=22.1$, $P<0.001$ and treatment group $\times$ day interaction effect: $F$ $(6,84)=11.8, P<0.0001)$, showing that the treatment of rats with this drug was capable of abolishing the expression of ADE. Calculations of alcohol consumption relative to baseline drinking demonstrated that effect of melatonin on post-abstinence drinking was similar to that of agomelatine (Supplementary Fig. 2). Fourier analysis of the melatonin treatment at the ZT12 data demonstrated that this treatment had a very similar effect on the phase shift to that of agomelatine. This treatment shifted the daily peak of ethanol drinking to earlier in the night. This observed phase shift was confirmed by Fourier calculations, showing significant overall phase advance (by $32 \mathrm{~min}, P<0.01$ ) in melatonintreated rats as compared with control vehicle-treated rats (Figure 2b, Table 2). In contrast, water intake was significantly increased in melatonin-treated animals during treatment days as compared with vehicle-treated rats (Figure 2c), suggesting that the effect of treatment was selective for ethanol (factor treatment group and treatment group $\times$ day interaction effect $\mathrm{F}(1,14)=23.9, P<0.001$ and $\mathrm{F}(6,84)=6.9, P<0.0001$, respectively). Similarly to ethanol, ZT12 melatonin administration shifted the water drinking peak to earlier in the night (by $3 \mathrm{~h} 11 \mathrm{~min}, P<0.05$ ) when compared with control vehicle-treated rats (Figure 2d, Table 2). It should be noted that melatonin treatment led to small but significant reduction of the locomotor activity during animals active phase (factor treatment group: $\mathrm{F}$ $(1,14)=4.9, P<0.05$; data not shown). This reduction was seen only during the first post-abstinence day. Melatonin treatment also had a small effect on animals' body weight, demonstrating altered food intake and/or metabolism during the treatment days (factor treatment group: $\mathrm{t}(14)=3.4$, $P<0.01$ ) (difference in the body weight of the vehicletreated animal group when compared before and after treatment was $+1.4 \pm 0.2 \%$ and of the $50 \mathrm{mg} / \mathrm{kg}$ of melatonintreated animal group $+0.1 \pm 0.3 \%$ ).

In contrast, melatonin treatment at ZT0 did not have a strong effect on the ADE. A two-way ANOVA revealed a general significant increase in total daily ethanol intake after a deprivation phase as compared to basal drinking (factor day: $\mathrm{F}(6,78)=43.9, P<0.0001)$. The drug treatment caused a small but significant reduction of the post-abstinence 

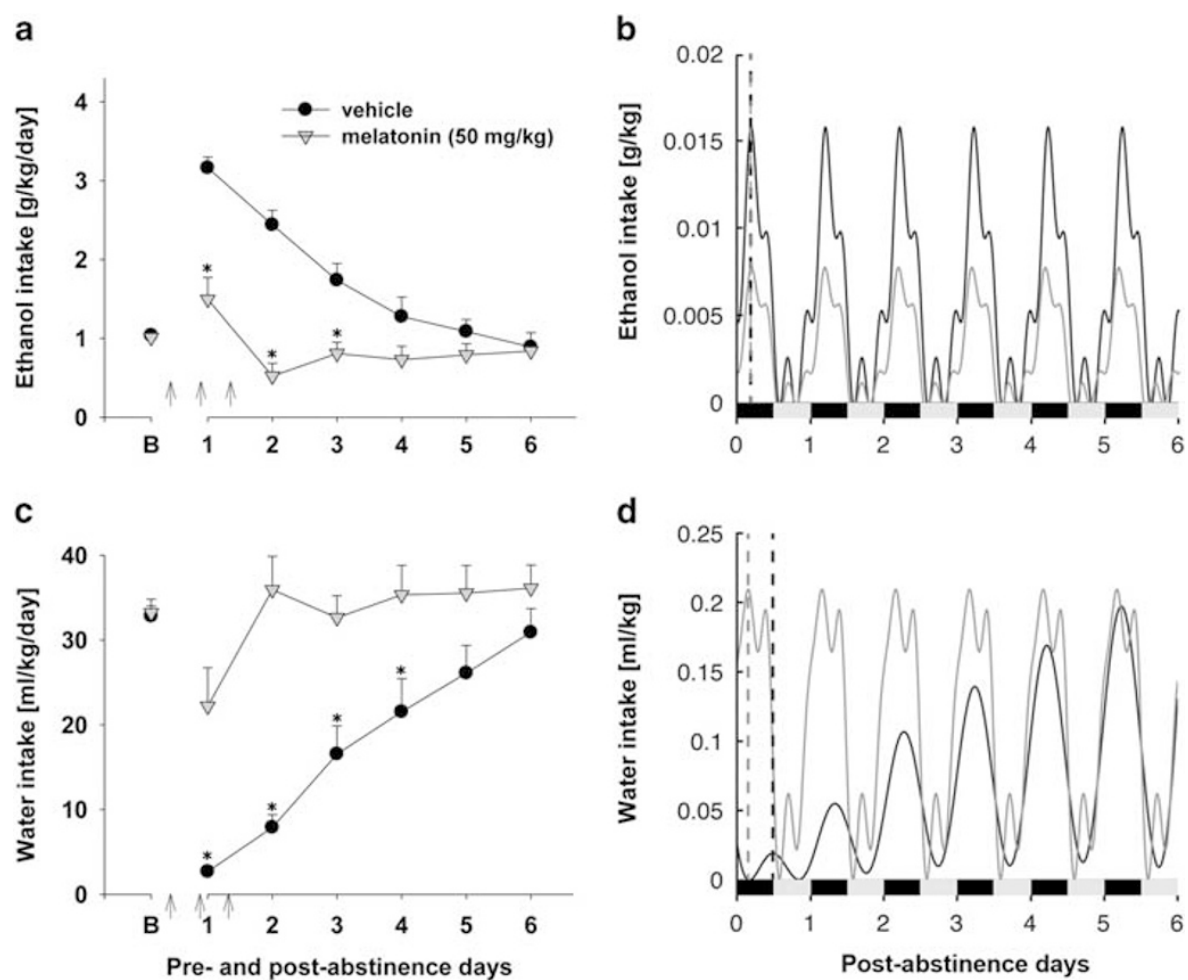

Figure 2 Total daily ethanol intake ( $\mathrm{g} / \mathrm{kg}$ per day) before and after an alcohol deprivation period of two weeks (a) and the Fourier representations of the averaged 5-min ethanol $(\mathrm{g} / \mathrm{kg}$ ) intake during the first six post-abstinence drinking days (b). Total daily water intake (ml/kg per day) before and after an alcohol deprivation period of two weeks (c) and the Fourier representations of the averaged 5-min water $(\mathrm{ml} / \mathrm{kg})$ intake during the first six post-abstinence drinking days (d). Arrows indicate the administration of either vehicle $(n=8)$ or $50 \mathrm{mg} / \mathrm{kg}$ of melatonin $(n=8)$. Drug administration was performed at ZT I2 (ie, at the end of the light phase). The last six days measurements of ethanol and water intake is given as baseline drinking-B. Data are presented as means \pm SEM *indicates significant differences from the vehicle treatment group, $P<0.05$. In (b) and (d) the vehicle-treated animal group is marked as a black line and the melatonin-treated animal group is marked as a gray line. Vertical dashed lines mark the peak fluid consumption within the first $24 \mathrm{~h}$ in vehicle (black) and melatonin (gray) treated animal groups. The black and gray bars on the $x$-axis indicate the dark and light phases, respectively.

drinking as compared with alcohol intake in vehicle-treated animals (factor treatment group $\times$ day interaction effect: $F$ $(6,78)=2.6, P<0.05)$. However the ADE was still present on the first post-abstinence day (Figure 3a). Subsequent post hoc analysis confirmed that total ethanol intake on the first ADE day was not significantly different between vehicle- and melatonin-treated rat groups. Fourier analysis showed that melatonin treatment at ZT0 shifted the peak of ethanol drinking to later in the night during the first ADE day. However, this observed phase shifting was very short lasting, as phase-shift calculations of post-abstinence ethanol drinking did not revealed any significant effect of ZT0 melatonin on the phase $(P=0.98$; Figure $3 \mathrm{~b}$, Table 2$)$. Water intake was not affected in ZT0 melatonin-treated animals when compared with vehicle-treated rats (factor treatment group and treatment group $\times$ day interaction effect $P=0.41$ and $P=0.10$, respectively; Figure $3 \mathrm{c}$ ). Similarly to ethanol, ZT0 melatonin administration had only very minor temporal phase shift to later in the night in case of water consumption when compared with control vehicle-treated rats $(P=0.96)$ (Figure 3d, Table 2). Locomotor activity measured during animals' active phase was not significantly affected by ZT0 melatonin treatment (factor treatment group and treatment group $\times$ day interaction effect $P=0.71$ and $P=0.73$, respectively; data not shown). Similarly, this treatment had no effect on animal's body weight $(P=0.45)$.
During SB242084 treatment a typical general increase in ethanol consumption was observed (factor day: $\mathrm{F}$ $(6,90)=37.7, P<0.0001)$. Even though SB242084 treatment caused significant reduction in total ethanol consumption when compared to that in vehicle-treated rats (factor treatment group: $\mathrm{F}(1,15)=6.3, P<0.05$ and a significant treatment group $\times$ day interaction effect: $\mathrm{F}(6,90)=4.5$, $P<0.001$ ), post hoc analysis demonstrated significant increase in ethanol intake during the first two post-abstinence drinking days in the drug-treated group as compared with the baseline consumption (Figure 4a). Fourier analysis of the data derived from SB242084 treatment did not show a phase shift in ethanol consumption, which was confirmed by phase-shift calculations (Figure 4b, Table 2). As a result of SB242084 treatment total daily water intake was significantly increased when compared with the vehicle treatment (factor treatment group $\times$ day interaction effect: $\mathrm{F}(6,90)=5.6$, $P<0.0001$ ) (Figure 4c). Interestingly, despite the lack of effect on ethanol drinking circadian phase, SB242084 treatment shifted water drinking peak to earlier in the night, which was confirmed by phase-shift calculations (by $2 \mathrm{~h}$ $26 \mathrm{~min}, P<0.01$; Figure 4d, Table 2). Neither locomotor activity nor body weight was significantly changed by SB242084 treatment (factor treatment group: $P=0.09$ and $P=0.50$ for locomotor activity and body weight, respectively; data not shown). 
a

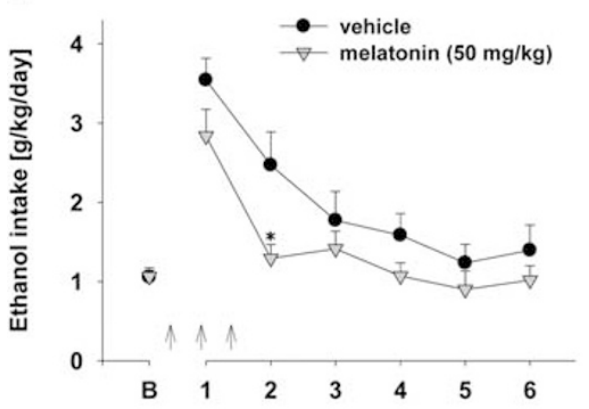

C

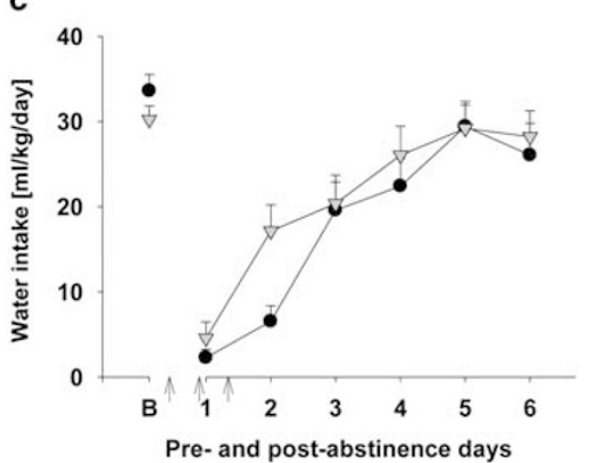

b

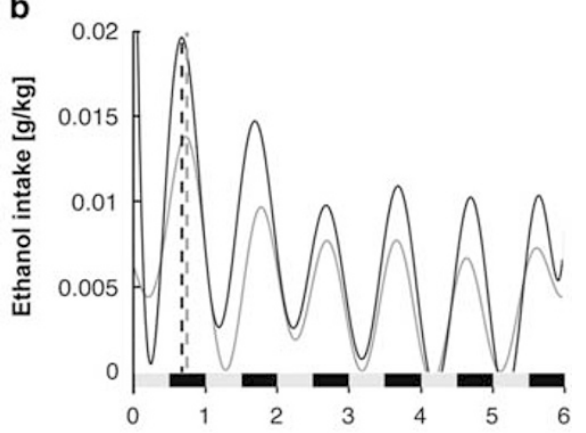

d

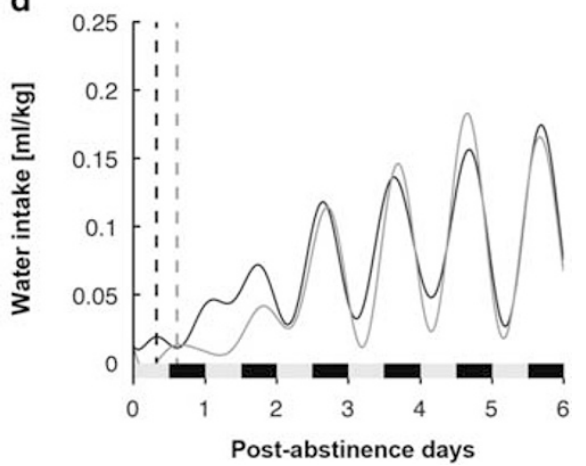

Figure 3 Total daily ethanol intake ( $g / k g$ per day) before and after an alcohol deprivation period of 2 weeks (a) and the Fourier representations of the averaged 5 -min ethanol $(\mathrm{g} / \mathrm{kg}$ ) intake during the first six post-abstinence drinking days (b). Total daily water intake (ml/kg per day) before and after an alcohol deprivation period of two weeks (c) and the Fourier representations of the averaged 5-min water $(\mathrm{ml} / \mathrm{kg})$ intake during the first six post-abstinence drinking days (d). Arrows indicate the administration of either vehicle $(n=7)$ or $50 \mathrm{mg} / \mathrm{kg}$ of melatonin $(n=8)$. Drug administration was performed at ZT0 (ie, at the onset of the light phase). The last 6 days measurements of ethanol and water intake is given as baseline drinking-B. Data are presented as means \pm SEM *indicates significant differences from the vehicle treatment group, $P<0.05$. In (b) and (d) the vehicle-treated animal group is marked as a black line and the melatonin-treated animal group is marked as a gray line. Vertical dashed lines mark the peak fluid consumption within the first $24 \mathrm{~h}$ in vehicle (black) and melatonin (gray) treated animal groups. The black and gray bars on the $x$-axis indicate the dark and light phases, respectively.

\section{DISCUSSION}

The present study demonstrates that all drug treatmentsagomelatine, melatonin, and $5-\mathrm{HT}_{2 \mathrm{C}}$ receptor antagonist SB242084, all given at the end of the light phase, as well as melatonin given at the onset of the light phase-reduced relapse-like alcohol intake in a four-bottle free choice paradigm in male Wistar rats. All four treatments tended or significantly increased water consumption during treatment days, suggesting the selectivity of these drugs toward alcohol. The treatments had no side effects, apart from melatonin given at the end of the light phase, which had minor effect on home cage locomotor activity and body weight. In summary, our study shows that the melatonergic system has a modulatory role on alcohol 'wanting' and relapse-like drinking behavior.

Both agomelatine and melatonin administered at the end of the light phase led to very similar changes on all measures of the post-abstinence drinking behavior, suggesting that effect of agomelatine on relapse-like behavior is mostly driven by its melatonergic activity. First of all, both of the treatments reduced the frequency of approaches to alcohol bottles to near that of baseline drinking, suggesting that they both had an effect on increased alcohol 'wanting', a characteristic of post-abstinence drinking behavior. An abstinence-induced increase in alcohol 'wanting' can be measured as a dramatically increased frequency of approaches to more concentrated alcohol solutions when compared to baseline drinking and a complete loss of normal diurnal patterns of consumption (Vengeliene et al, 2013). Interestingly, consumed amount of more concentrated alcohol solutions per drinking approach was not affected by either agomelatine or melatonin treatment, which could be interpreted in a way that these treatments had no effect on alcohol 'liking' (Vengeliene et al, 2013). And finally both agomelatine and melatonin given at the end of the light phase caused a significant circadian phase advance in the diurnal drinking of both water and alcohol when compared to the control vehicle-treated rats. Similar behavioral responses to these compounds could be expected. Although interaction of agomelatine with $5-\mathrm{HT}_{2 \mathrm{C}}$ receptors distinguishes this compound from melatonin, affinity of agomelatine for this serotonergic receptor is much lower than its affinity for the melatonergic MT1 and MT2 receptors (Millan et al, 2003; Hardeland, 2009). Accordingly, disturbances of circadian rhythmicity were shown to be affected in a similar manner by administration of either agomelatine or melatonin (Van Reeth et al, 2001).

Nevertheless, different pharmacological profiles of agomelatine and melatonin should be taken into account. Earlier studies demonstrated that high doses of these two compounds have divergent effects on brain activity-administration of agomelatine elevated extracellular levels of dopamine (DA) and norepinephrine $(\mathrm{NE})$ in the frontal 

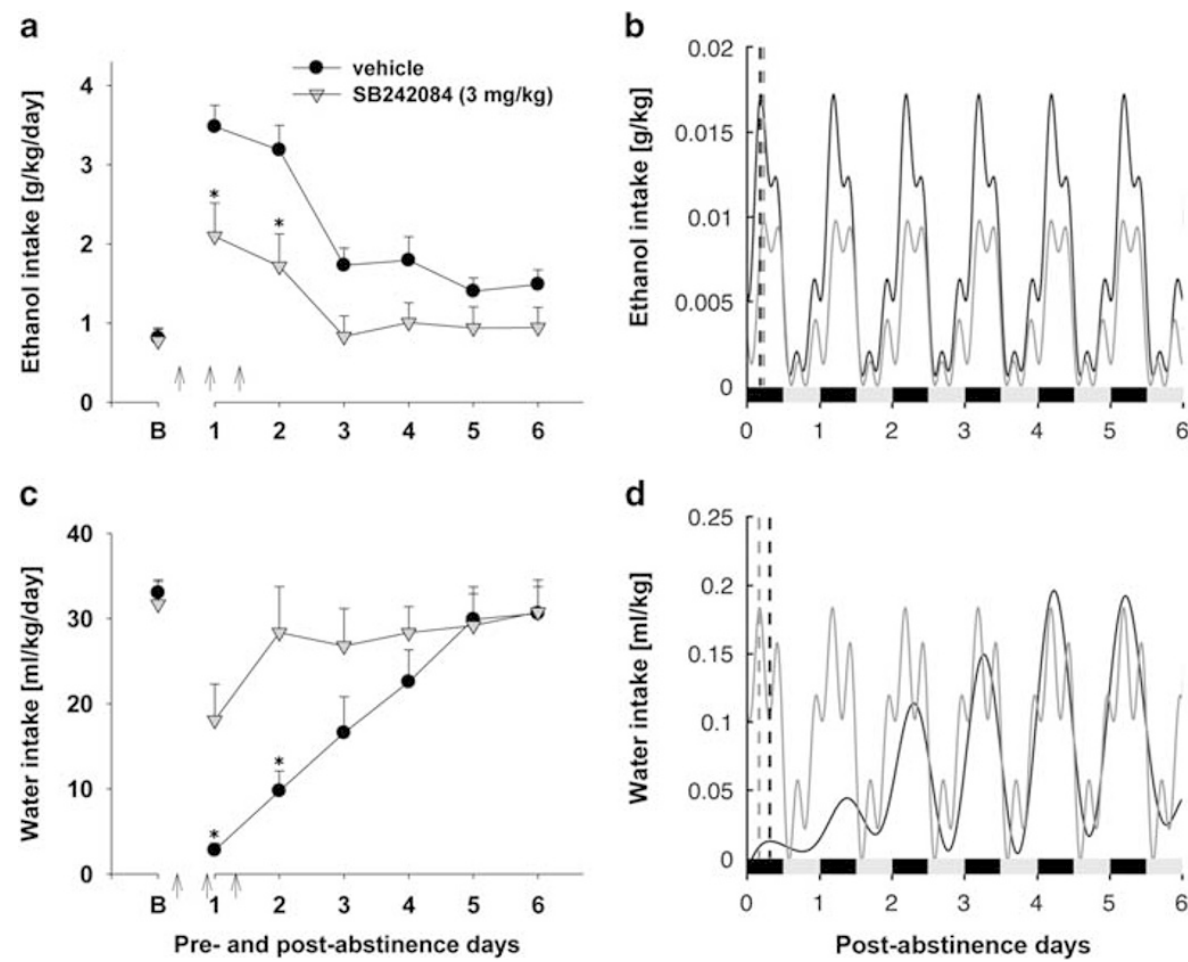

Figure 4 Total daily ethanol intake ( $g / k g$ per day) before and after an alcohol deprivation period of 2 weeks (a) and the Fourier representations of the averaged 5-min ethanol ( $\mathrm{g} / \mathrm{kg}$ ) intake during the first six post-abstinence drinking days (b). Total daily water intake (ml/kg per day) before and after an alcohol deprivation period of 2 weeks (c) and the Fourier representations of the averaged 5-min water $(\mathrm{ml} / \mathrm{kg})$ intake during the first six post-abstinence drinking days (d). Arrows indicate the administration of either vehicle $(n=8)$ or $3 \mathrm{mg} / \mathrm{kg}$ of SB242084 (n=9). Drug administration was performed at ZT I2 (ie, at the end of the light phase). The last six days measurements of ethanol and water intake is given as baseline drinking-B. Data are presented as means \pm SEM $*$ indicates significant differences from the vehicle treatment group, $P<0.05$. In (b) and (d) the vehicle-treated animal group is marked as a black line and the SB242084 treated animal group is marked as a gray line. Vertical dashed lines mark the peak fluid consumption within the first $24 \mathrm{~h}$ in vehicle (black) and SB242084 (gray) treated animal groups. The black and gray bars on the $x$-axis indicate the dark and light phases, respectively.

cortex of freely moving rats, while melatonin did not have significant effect on these neurotransmitters (Millan et al, 2003). As said differences between agomelatine and melatonin are mostly attributed to the effect of agomelatine on the $5-\mathrm{HT}_{2 \mathrm{C}}$ receptor, since the selective antagonist of this receptor SB242084 increases-similar to agomelatine-levels of DA and NA in the frontal cortex (Gobert et al, 2000). In other studies administration of either agomelatine or combination of melatonin and SB242084 was shown to increase the number of spontaneously active DA neurons within the ventral tegmental area, whereas administration of melatonin alone had no effect on activity of these neurons (Chenu et al, 2013; 2014). Although in the present study we used a relatively high dose of agomelatine our comparative analysis suggest only a minor if at all contribution of $5 \mathrm{HT}_{2 \mathrm{C}}$ receptors in mediating the effects on alcohol 'wanting' and relapse-like drinking behavior. Thus, in our study the $5 \mathrm{HT}_{2 \mathrm{C}}$ antagonist SB242084 did not have the same effect on drinking behavior as agomelatine. Its effect on drinking frequency and average intake during a drinking approach was not consistent with the pattern produced by agomelatine treatment. It did reduce drinking frequency of all alcohol solutions but, contrary to agomelatine, intake of $20 \%$ alcohol per drinking approach was even increased. This finding is not surprising, as it was reported earlier that SB242084 increases operant responding for alcohol (Tomkins et al, 2002). Most importantly, our study demonstrated that SB242084 had no effect on circadian phase of alcohol consumption, although it caused a significant phase advance in case of water consumption. We therefore conclude that the effects of agomelatine on alcohol 'wanting' and relapselike drinking behavior are mediated via MT1/2 receptors. As already mentioned, melatonergic receptors were found in several dopamine innervated structures, such as prefrontal cortex, striatum, nucleus accumbens and amygdala ( $\mathrm{Uz}$ et al, 2005). Administration of physiological doses of melatonin in rats was shown to increase the affinity of the D2 dopamine receptors (Hamdi, 1998) and generally produce antidopaminergic effect on the brain (for review see Zisapel, 2001). Although high doses of melatonin have different effect on the dopaminergic system (Millan et al, 2003), the reduced 'wanting' is likely attributable to the indirect effect of our treatments with this system.

Sleep disturbance even after months of abstinence in alcohol-dependent people are likely caused by lower levels of melatonin and delayed peak of its nocturnal rise (Drummond et al, 1998; Kühlwein et al, 2003). A very similar pattern of melatonin levels was also demonstrated in alcohol drinking rats (Peres et al, 2011). In our study, melatonin given at the onset of the light phase reduced alcohol intake but this reduction was somewhat weak-the $\mathrm{ADE}$ was not significantly different from that of the control animals on the first post-abstinence day. On this day relapselike behavior is the most pronounced. Above all, melatonin given at the onset of the light phase had no effect on the circadian phase of either alcohol or water drinking. This data 
shows the importance of circadian phase advancement on alcohol consumption, as administration of melatonin at the end of the light phase advanced circadian phase and completely abolished ADE.

Contrary to our findings, earlier studies have shown that administration of very low doses of melatonin $(0.2-5 \mathrm{mg} / \mathrm{kg})$ had opposite effects on alcohol consumption and self-administration (Geller, 1971; Smith et al, 1980; Crespi, 2012). We used much higher doses of melatonin in our study, which have different effects on brain function. For instance, it was demonstrated that at low doses $(0.5-1 \mathrm{mg} / \mathrm{kg})$ melatonin decreased firing activity of dorsal raphe nucleus 5-HT neurons (Domínguez-López et al, 2012) while administration of high doses of melatonin (up to $40 \mathrm{mg} / \mathrm{kg}$ ) had no such effect (Domínguez-López et al, 2012; Chenu et al, 2014). The other important difference of our study was that melatonin was tested under relapse conditions, which might involve different brain structures and different neurotransmission than drinking under baseline conditions (Vengeliene et al, 2008; Spanagel, 2009).

Currently, there are four antirelapse medications approved by the US Food and Drug Administration (FDA) to treat alcohol dependence: disulfiram, oral naltrexone, a longlasting injectable naltrexone and acamprosate (Litten et al, 2012; for the later drug see also Spanagel et al, 2014). These drugs have small to medium effect sizes and only a minority of the patients do benefit from these treatments (Litten et al, 2012). Another problem is that there is a huge treatment gap -in fact only $10 \%$ of the alcoholics obtain an appropriate treatment. Given this situation new drugs that can be easily translated into the clinical situation are warranted. One of those candidates is agomelatine since it is already approved and clinically used as an antidepressant in many countries (Gahr, 2014; Taylor et al, 2014). Given that there is a high comorbidity between alcoholism and depressive behavior general practitioners can easily prescribe agomelatine for this comorbid condition. In conclusion, targeting the melatonergic system with agomelatine (or melatonin) in patients suffering from alcoholism, especially those with comorbid depression, may help to restore normal sleep architecture of an addicted patient, reduce alcohol wanting and thereby relapse behavior.

\section{FUNDING AND DISCLOSURE}

This work was supported by the Bundesministerium für Bildung und Forschung (e:Med program; FKZ: 01ZX1311A (Spanagel et al, 2013)). RS disclosed having received honoraria from Servier, Germany. The authors declare no conflict of interest, financial or otherwise.

\section{ACKNOWLEDGMENTS}

We thank Sabrina Koch for her technical assistance.

\section{REFERENCES}

Brick J, Pohorecky LA, Faulkner W, Adams MN (1984). Circadian variations in behavioral and biological sensitivity to ethanol. Alcohol Clin Exp Res 8: 204-211.
Chenu F, El Mansari M, Blier P (2013). Electrophysiological effects of repeated administration of agomelatine on the dopamine, norepinephrine, and serotonin systems in the rat brain. Neuropsychopharmacology 38: 275-284.

Chenu F, Shim S, El Mansari M, Blier P (2014). Role of melatonin, serotonin $2 \mathrm{~B}$, and serotonin $2 \mathrm{C}$ receptors in modulating the firing activity of rat dopamine neurons. J Psychopharmacol 28: 162-167.

Crespi F (2012). Influence of melatonin or its antagonism on alcohol consumption in ethanol drinking rats: a behavioral and in vivo voltammetric study. Brain Res 1452: 39-46.

Domínguez-López S, Mahar I, Bambico FR, Labonté B, Ochoa-Sánchez R, Leyton $M$ et al (2012). Short-term effects of melatonin and pinealectomy on serotonergic neuronal activity across the light-dark cycle. J Psychopharmacol 26: 830-844.

Drummond SP, Gillin JC, Smith TL, DeModena A (1998). The sleep of abstinent pure primary alcoholic patients: natural course and relationship to relapse. Alcohol Clin Exp Res 22: 1796-1802.

Gahr M (2014). Agomelatine in the treatment of major depressive disorder: an assessment of benefits and risks. Curr Neuropharmacol 12: 287-398.

Geller I (1971). Ethanol preference in the rat as a function of photoperiod. Science 173: 456-459.

Gobert A, Rivet JM, Lejeune F, Newman-Tancredi A, AdhumeauAuclair A, Nicolas JP et al (2000). Serotonin(2C) receptors tonically suppress the activity of mesocortical dopaminergic and adrenergic, but not serotonergic, pathways: a combined dialysis and electrophysiological analysis in the rat. Synapse 36: 205-221.

Hamdi A (1998). Melatonin administration increases the affinity of D2 dopamine receptors in the rat striatum. Life Sci 63: 2115-2120.

Hardeland R (2009). New approaches in the management of insomnia: weighing the advantages of prolonged-release melatonin and synthetic melatoninergic agonists. Neuropsychiatr Dis Treat 5: 341-354.

Kühlwein E, Hauger RL, Irwin MR (2003). Abnormal nocturnal melatonin secretion and disordered sleep in abstinent alcoholics. Biol Psychiatry 54: 1437-1443.

Lê A, Shaham Y (2002). Neurobiology of relapse to alcohol in rats. Pharmacol Ther 94: 137-156.

Litten RZ, Egli M, Heilig M, Cui C, Fertig JB, Ryan ML et al (2012). Medications development to treat alcohol dependence: a vision for the next decade. Addict Biol 17: 513-527.

Lynch HJ, Ozaki Y, Shakal D, Wurtman RJ (1975). Melatonin excretion of man and rats: effect of time of day, sleep, pinealectomy and food consumption. Int J Biometeorol 19: 267-279.

Millan MJ, Gobert A, Lejeune F, Dekeyne A, Newman-Tancredi A, Pasteau V et al (2003). The novel melatonin agonist agomelatine (S20098) is an antagonist at 5-hydroxytryptamine2C receptors, blockade of which enhances the activity of frontocortical dopaminergic and adrenergic pathways. J Pharmacol Exp Ther 306: 954-964.

Noori HR, Spanagel R, Hansson AC (2012). Neurocircuitry for modeling drug effects. Addict Biol 17: 827-864.

Perreau-Lenz S, Vengeliene V, Noori HR, Merlo-Pich EV, Corsi MA, Corti C et al (2012). Inhibition of the casein-kinase$1-\varepsilon / \delta /$ prevents relapse-like alcohol drinking. Neuropsychopharmacology 37: 2121-2131.

Peres R, do Amaral FG, Madrigrano TC, Scialfa JH, Bordin S, Afeche SC et al (2011). Ethanol consumption and pineal melatonin daily profile in rats. Addict Biol 16: 580-590.

Rhodes JS, Best K, Belknap JK, Finn DA, Crabbe JC (2005). Evaluation of a simple model of ethanol drinking to intoxication in C57BL/6J mice. Physiol Behav 84: 53-63.

Sinclair JD, Senter RJ (1967). Increased preference for ethanol in rats following alcohol deprivation. Psychonom Sci 8: 11-12.

Smith D, Oei TP, Ng KT, Armstrong S (1980). Rat selfadministration of ethanol: enhancement by darkness and exogenous melatonin. Physiol Behav 25: 449-455. 
Spanagel R (2009). Alcoholism: a systems approach from molecular physiology to addictive behavior. Physiol Rev 89: 649-705.

Spanagel R, Durstewitz D, Hansson A, Heinz A, Kiefer F, Köhr G et al (2013). A systems medicine research approach for studying alcohol addiction. Addict Biol 18: 883-896.

Spanagel R, Hölter SM (1999). Long-term alcohol selfadministration with repeated alcohol deprivation phases: an animal model of alcoholism? Alcohol Alcohol 34: 231-243.

Spanagel R, Vengeliene V, Jandeleit B, Fischer WN, Grindstaff K, Zhang X et al (2014). Acamprosate produces its anti-relapse effects via calcium. Neuropsychopharmacology 39: 783-791.

Taylor D, Sparshatt A, Varma S, Olofinjana O (2014). Antidepressant efficacy of agomelatine: meta-analysis of published and unpublished studies. BMJ 348: g1888.

Tomkins DM, Joharchi N, Tampakeras M, Martin JR, Wichmann J, Higgins GA (2002). An investigation of the role of 5-HT(2C) receptors in modifying ethanol self-administration behaviour. Pharmacol Biochem Behav 71: 735-744.

Uz T, Arslan AD, Kurtuncu M, Imbesi M, Akhisaroglu M, Dwivedi $\mathrm{Y}$ et al (2005). The regional and cellular expression profile of the melatonin receptor MT1 in the central dopaminergic system. Mol Brain Res 136: 45-53.

Van Reeth O, Weibel L, Olivares E, Maccari S, Mocaer E, Turek FW (2001). Melatonin or a melatonin agonist corrects age-related changes in circadian response to environmental stimulus. Am J Physiol Regul Integr Comp Physiol 280: R1582-R1591.

Vengeliene V, Bilbao A, Molander A, Spanagel R (2008). Neuropharmacology of alcohol addiction. Br J Pharmacol 154: 299-315.

Vengeliene V, Bilbao A, Spanagel R (2014). The alcohol deprivation effect model for studying relapse behavior: a comparison between rats and mice. Alcohol 48: 313-320.

Vengeliene V, Noori HR, Spanagel R (2013). The use of a novel drinkometer system for assessing pharmacological treatment effects on ethanol consumption in rats. Alcohol Clin Exp Res 37: E322-E328.

von Gall C, Stehle JH, Weaver DR (2002). Mammalian melatonin receptors: molecular biology and signal transduction. Cell Tissue Res 309: 151-162.

Zisapel N (2001). Melatonin-dopamine interactions: from basic neurochemistry to a clinical setting. Cell Mol Neurobiol 21: 605-616.

Supplementary Information accompanies the paper on the Neuropsychopharmacology website (http://www.nature.com/npp) 